\title{
La visión parkinsoniana de la figura compleja de Rey-Osterrieth
}

\author{
E. Noé-Sebastián, E. Martínez-Vila, M.'R. Luquin \\ THE PARKINSONIAN VIEW OF THE REY-OSTERRIETH COMPLEX FIGURE
}

Summary. Visuospatial impairment has been frequently reported in Parkinson's disease (PD). We present the progressive distorsioned performance of the Rey-Osterrieth complex figure in parkinsonian patients at different stages of the disease (PD de novo, PD on long-term treatment, PD with phychosis and PD with dementia) [REV NEUROL 1999; 28: 434-5].

Key words. Parkinson'disease. Psychology. Rey-Osterrieth complex figure. Visuospatial.

El test de la figura compleja de Rey-Osterrieth, elaborado por A. Rey y P.A. Osterrieth entre 1941 y 1944, permite estudiar una amplia variedad de procesos cognitivos [1]. La ejecución de este test consiste en solicitar al sujeto examinado que copie una figura compleja a mano y sin límite de tiempo, valorándose la capacidad de organización y planificación de estrategias para la resolución de problemas así como su capacidad visuoconstructiva. Posteriormente, sin previo aviso y sin la ayuda del modelo, el examinado debe reproducir inmediatamente y a los 30 minutos de nuevo la misma figura, con el fin de evaluar su capacidad de recuerdo de

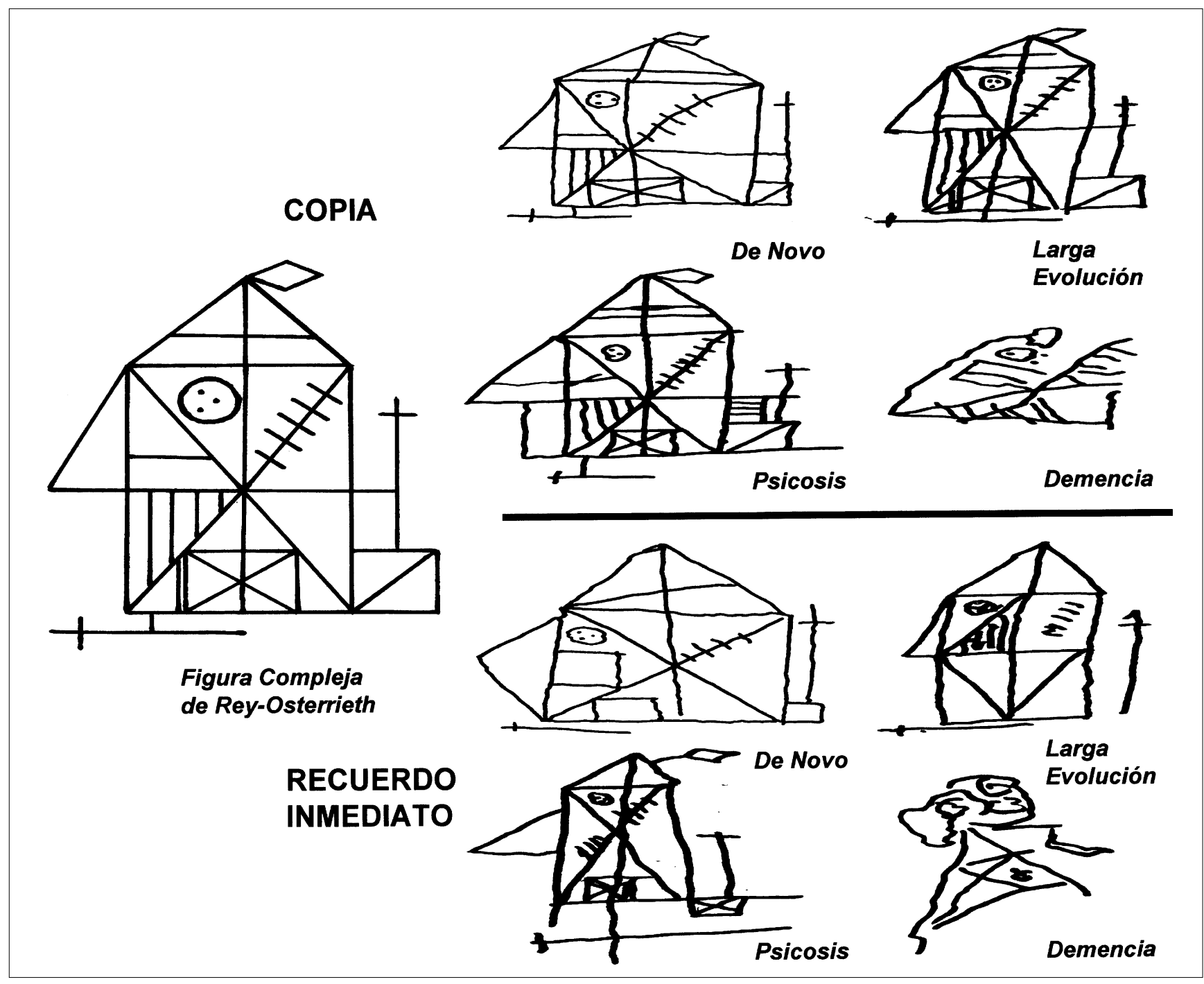

Recibido:31.08.98. Aceptadotras revisiónexterna sinmodificaciones: 12.09.98.

Departamento de Neurología. Clínica Universitaria de Navarra. Pamplona, España.
Correspondencia: Dr. Enrique Noé Sebastián. Departamento de Neurología. Clínica Universitaria de Navarra. Avda. Pío XII, s/n. E-31080 Pamplona. E-mail: enoesebas@unav.es

(C) 1999, REVISTA DE NEUROLOGÍA 
material no verbal. Cada uno de estos intentos se puntúa sobre una escala validada que oscila desde un mínimo de 0 a un máximo de 36 puntos (normalidad).

Las alteraciones en labores visuoespaciales constituye uno de los déficit cognitivos más frecuentemente demostrados en pacientes con enfermedad de Parkinson (EP) [2]. Hemos utilizado este sencillo test para evaluar las habilidades visuoconstructivas en pacientes con EP en distintos estadios clínicos. La imagen muestra la correcta ejecución de este test en pacientes con EP de novo, así como la progresiva desestructuración de la copia y recuerdo de la figura en pacientes de larga evolución y en pacientes con complicaciones psiquiátricas, hasta su completa distorsión haciéndose irreconocible en los pacientes con EP y demencia. Recientemente se ha señalado que el déficit visuoespacial en pacientes con EP es producto de una disminución en lacapacidad de manejar información no verbal, producto de la disfunción del circuito estriado-talamocortical que incluiría la corteza prefrontal dorsolateral y también la corteza inferotemporal encargada de procesar material visuoespacial $[3,4]$.

\section{BIBLIOGRAFÍA}

1. Strauss E, Spreen O. A compendium of neuropsychological tests. Administration, Norms, and Commentary. 2 ed. New York: Oxford University Press; 1998.

2. Levin BE, Llabre MM, Reisman S, Weiner WJ, Sánchez-Ramos J, Singer C, et al. Visuospatial impairment in Parkinson's disease. Neurology 1991; 3: 365-9.

\section{LA VISIÓN PARKINSONIANA DE LA FIGURA COMPLEJA DE REY-OSTERRIETH}

Resumen. Los pacientes con enfermedad de Parkinson (EP) padecen frecuentemente alteraciones en tareas visuoespaciales. Presentamos la aplicación del test de la figura compleja de Rey-Osterrieth en pacientes con distintos estadios de EP (EP de novo, EP de largo tratamiento, EP con psicosis y EP con demencia) [REV NEUROL 1999; 28:].

Palabras clave. Enfermedad de Parkinson. Figura compleja de ReyOsterrieth. Psicología. Visuoespacial.
3. Dubois B, Pillon B. Cognitive deficits in Parkinson's disease. J Neurol 1997; 1: 2-8.

4. Middleton FA, Strick PL. The temporal lobe is a target of output from the basal ganglia. Proc Natl Acad Sci U S A 1996; 16: 8683-7.

\section{A VISÃO PARKINSÓNICA DA FIGURA COMPLEXA DE REY-OSTERRIETH}

Resumo. Os doentes com doença de Parkinson (DP) têm frequentemente alterações nas tarefas visuoespaciais. Apresentamos a aplicação do teste da figura complexa de Rey-Osterrieth em doentes com diferentes estadios de DP (DP de novo, DP de longo tratamento, $D P$ com psicose e DP com demência) [REV NEUROL 1999; 28:].

Palavras chave. Doença de Parkinson. Figura complexa de ReyOsterrieth. Psicologia. Visuoespacial. 